\title{
The surface of asteroid Bennu
}

\section{$c 4$}

We expected

a young and

refreshed

surface, but

found many

very old

features on

the surface

of Bennu

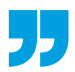

Asteroids are composed of remnant material from the early solar system that was not processed into planets. Therefore, they offer a window into the building blocks of the solar system, the composition of the solar nebula and the origins of life. The near Earth asteroid (NEA) 101955 Bennu is of particular interest owing to its presumed organic-rich composition, potential economic resources and the high probability of it impacting Earth towards the end of the 22nd century.

The first data on the surface composition and structure of asteroid Bennu has been obtained by the Origins, Spectral Interpretation, Resource Identification, SecurityRegolith Explorer (OSIRIS-REx) mission, which entered into orbit around Bennu in December 2018. Over the following year, the explorer will continue to map the asteroid and gather spectroscopic data, before contacting the asteroid

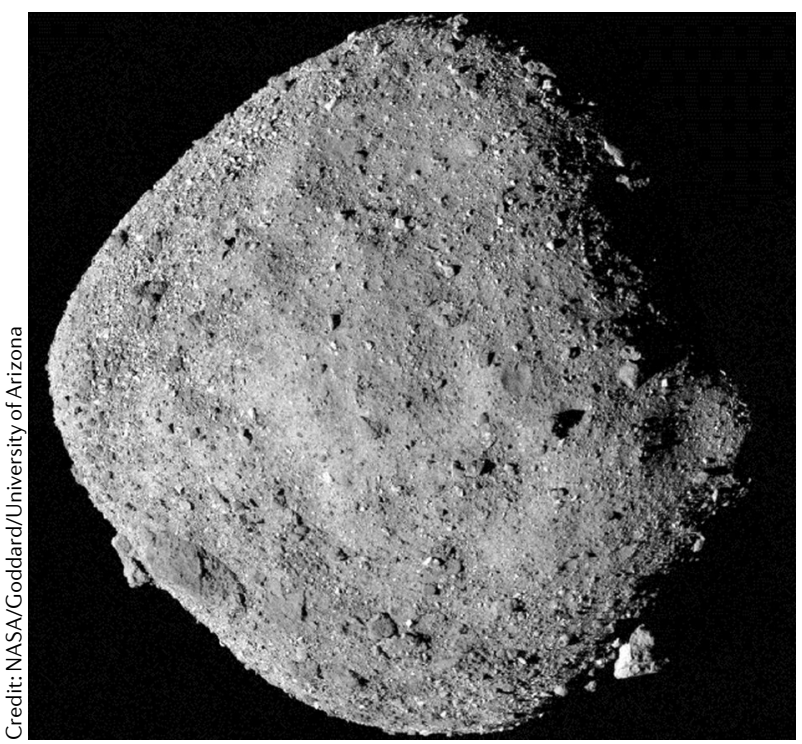

briefly in 2020 to collect sample material and returning to Earth by 2023. The surface evolution and composition of Bennu have now been reported in Nature Geoscience and Nature Astronomy, respectively, revealing that Bennu's surface is unexpectedly old and hydrated.

Writing in Nature Geoscience, Kevin Walsh and colleagues estimated the surface evolution and age of the asteroid. Structurally, Bennu is characterized as a rubble pile - "an unconsolidated asteroid held together by its own gravity that formed from re-accumulated impact debris in the asteroid belt," explains Walsh. By assessing Bennu's largest craters, the researchers calculate a surface age of one hundred million to one billion years. This old age, reflecting Bennu's departure date from the asteroid belt, is surprising for NEAs, which are expected to have young surface ages of about ten million years owing to weathering by solar heating and gravitational effects of planetary fly-bys. "We expected a young and refreshed surface, but found many very old features on the surface of Bennu," comments Walsh. Nevertheless, the team also report some evidence for more recent surface processes, such as localized movement of boulders and infilling of craters with weathered material.

Reporting in Nature Astronomy, Victoria Hamilton and colleagues evaluated spectroscopic data to confirm ground-based observations that Bennu exhibits a uniform, blue (negative) spectral slope. "The principal result so far," summarizes Hamilton, "is that Bennu is comprised of a large fraction of water-bearing minerals," suggesting that Bennu has a similar composition to aqueously altered carbonaceous chondrites. "This composition indicates that Bennu's parent body underwent significant interaction with water during its life in the asteroid belt," says Hamilton.

The spectral data reveal that Bennu's surface is rich in hydrated iron-rich clay minerals, and contains magnetite $\left(\mathrm{Fe}_{3} \mathrm{O}_{4}\right)$ - a composition that may have been derived from the weathering of Bennu's boulders. The authors suggest that objects like Bennu could represent a class of asteroids responsible for delivering volatile species and possibly organics to the early Earth.

These results represent the initial fruits of a 2-year interaction with Bennu, which will culminate in a touch-and-go sample retrieval mission. The researchers anticipate that future, higher-resolution observations and the analysis of the returned sample will answer many outstanding questions about the formation and evolution of Bennu, as well as the history of water and organics in the early solar system.

Tom Henshall

ORIGINAL ARTICLES Walsh, K. J. et al. Craters, boulders and regolith of (101955) Bennu indicative of an old and dynamic surface. Nat. Geosci. https://doi.org/10.1038/s41561-019-0326-6 (2019) |Hamilton, V. E. et al. Evidence for widespread hydrated minerals on asteroid (101955) Bennu. Nat. Astron. https://doi. org/10.1038/s41550-019-0722-2 (2019) FURTHER READING Scheeres, D. J. et al. The dynamic geophysical environment of (101955) Bennu based on OSIRIS-Rex measurements. Nat. Astron. https://doi.org/10.1038/s41550-0190721-3 (2019) |Barnouin, O. S. et al. Shape of (101955) Bennu indicative of a rubble pile with internal stiffness. Nat. Geosci. https://doi.org/ 10.1038/s41561-019-0330-x (2019) 